\title{
Reduced Order Model and Control of Non-Isolated High Gain Boost Converter
}

\author{
M. Muhammad, M. Armstrong, M. A. Elgendy, S. Lambert, V. Pickert \\ School of Electrical Electronic Engineering Newcastle University \\ Newcastle upon Tyne, United Kingdom \\ m.muhammad@ncl.ac.uk; matthew.armstrong@ncl.ac.uk; mohammed.elgendy@newcastle.ac.uk
}

\begin{abstract}
This paper present a reduced order model of interleaved boost converter integrating coupled inductor and switched capacitor by decoupling the leakage inductance leading to resonant exchange with the switched capacitors. State space averaging method is employed to derive the small signal ac model with ideal components. Furthermore, a dual loop control is adopted to regulate the output voltage of the converter. Extensive analysis and simulation demonstrate that the proposed model although simplified is sufficient for an adequate control system design ensuring fast transient response and good output voltage regulation.
\end{abstract}

Keywords-High step-up DC-DC Converter, reduced order modelling, Control design, Coupled Inductor, Switched capacitor.

\section{INTRODUCTION}

Most of the devices that produce or store electrical energy (e.g. batteries, ultra-capacitors, fuel cells and solar photovoltaic PV) are built from low voltage sources which are usually connected in series to achieve desired voltage. Series connection of cells increases the system complexity and reduced performance due to manufacturing variation among cells and different working conditions. In addition, these sources also have a significant variation of their output voltages due to different state of charge (SoC) or the solar radiation [1].

Typical applications requires converters with high voltage gain, usually ten times or higher to harvest all available energy, which calls for high efficiency. These features have been the focus of many researches and huge number of topologies have proposed to this end.

Great effort has been devoted to dc-dc converter topologies fed by renewable sources, however, only few works have been found in modeling these converters. A dynamic model of six phase interleaved double dual boost and its control design is presented in [2]. Model investigation of three switching cell boost converter is described in [3], and the small signal model of current fed converter is presented in [4]. A full and reduced order model for the dc-dc multilevel boost converter based on state space averaging are proposed in [5]. The main reason being that most of the topologies have resonant stages or ripple that cannot be neglected in one or more state variables, which is key to requirement in applying state space averaging method.

This paper explore a topology proposed with the objective of realizing higher voltage gain in comparison with the conventional interleaved boost converter [6]. An interesting feature is that the power devices can be sized to a voltage lower than the output voltage. The main objective of this paper is to propose a reduced order model by excluding the leakage inductance of the coupled inductor which is inherent phenomena of the coupled inductor [1], [6], [7]. The paper presents a linearized dynamic model of interleaved boost converter integrating coupled inductor and switched capacitor. The result shows that the model differ from that of conventional interleaved couple inductor boost converter and the reduced order model is a good approximation of the system behavior.

\section{CONVERTER DESCRIPTION AND ANALYSIS}

Fig. 1 shows the two phase interleaved high step-up converter, where $R_{O}$ represent the load and $V_{i n}$ and $V_{O}$ represent the input and output voltages respectively. Each of the converter phase employs a coupled inductors and its corresponding pair of switch, clamp capacitor e.g. phase 1 comprises of coupled inductors $L_{1}$ switch $S_{1}$ clamp switch $S_{C 1}$.and clamp capacitor $C_{C 1}$. The primary winding of each coupled inductor e.g. $L_{1 a}$ is coupled to the corresponding secondary windings $L_{1 b}$. The primary and secondary windings are denoted by $n_{1}, n_{2}$, and the coupling references denoted by "o" and "*'. $L_{m}$ denotes the magnetizing inductance of the coupled inductors whilst $L_{K}$ represent the leakage inductances of the coupled inductors reflected to the secondary side. $C_{O}$ denotes the output capacitor.

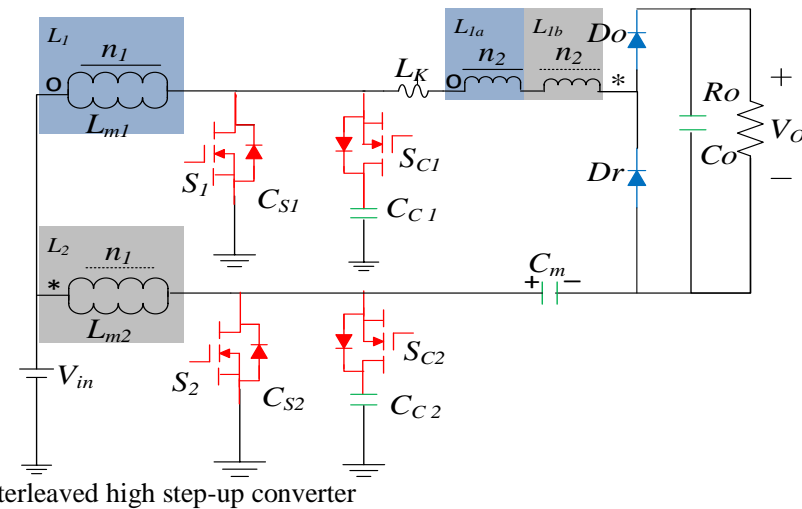

Fig. 1 Interleaved high step-up converter

During either of the main switch OFF instant, the voltage across the clamp and capacitors is denoted by

$$
V_{C c 1}=V_{C c 2}=V_{\text {in }} /(1-D)
$$

When the power switch $S_{1}$ turns OFF the voltage across the switched capacitor and output voltage of the converter are 


$$
\begin{gathered}
V_{C m}=V_{O} / 2 \\
V_{O}=V_{C c 1}+N V_{i n}+N\left(V_{C c 1}-V_{i n}\right)+V_{C m}
\end{gathered}
$$

Substituting (1) and (2) into (3), the voltage gain of the converter can be expressed as

$$
M=\frac{V_{O}}{V_{\text {in }}}=\frac{2 N+2}{(1-D)}
$$

Where $D$ is the converter duty is cycle and $N$ is the coupled inductor turns ratio.

\section{SMALL SIGNAL MODELLING}

A typical interleaved converter operation is divided into four subintervals (switch ON and OFF instances). During each interval, the converter equations can be written in state space model of the form

$$
\dot{x}= \begin{cases}A_{1} \mathbf{x}+B_{1} \mathbf{u} & S_{1} \text { and } S_{2} \text { on } \\ A_{2} \mathbf{x}+B_{2} \mathbf{u} & S_{1} \text { on } S_{2} \text { off } \\ A_{3} \mathbf{x}+B_{3} \mathbf{u} & S_{1} \text { on } S_{2} \text { on } \\ A_{4} \mathbf{x}+B_{4} \mathbf{u} & S_{1} \text { off } S_{2} \text { on }\end{cases}
$$

The state vector is defined in (6) by excluding the leakage inductance $L_{K}$ to attain the average model and considering $v_{C m}$ as constant voltage source described by (2)

$$
x=\left[\begin{array}{lllll}
i_{L m 1} & i_{L m 2} & v_{C c 1} & v_{C c 2} & v_{O}
\end{array}\right]^{T}
$$

and the input is defined as

$$
u=\left[V_{\text {in }}\right]
$$

Now assuming that the converter operate in continuous conduction (CCM) mode, no parasitic effect (i.e. neglecting short operating intervals), and switching frequency is much higher than the converter natural frequency. Equation (8)-(9)

$$
\begin{aligned}
& \left\{\begin{array}{c}
L_{m 1} \frac{d i_{L m 1}}{d t}=v_{i n} \\
L_{m 2} \frac{d i_{L m 2}}{d t}=v_{i n} \\
C_{O} \frac{d v_{o}}{d t}-\frac{v_{o}}{R_{O}}
\end{array}\right. \\
& \left\{\begin{array}{c}
L_{m 1} \frac{d i_{L m 1}}{d t}=v_{i n} \\
L_{m 2} \frac{d i_{L m 2}}{d t}=v_{i n}-v_{C c 2} \\
v_{C c 2}=\frac{v_{o}}{2}+N L_{m 2} \frac{d i_{L m 2}}{d t}-N v_{i n} \\
C_{O} \frac{d v_{o}}{d t}=-\frac{v_{o}}{R_{O}}
\end{array}\right. \\
& \left\{\begin{array}{c}
L_{m 1} \frac{d i_{L m 1}}{d t}=v_{i n}-v_{C c 1} \\
L_{m 2} \frac{d i_{L m 2}}{d t}=v_{i n} \\
v_{C c 1}=\frac{v_{o}}{2}+N L_{m 1} \frac{d i_{L m 1}}{d t}-N v_{i n} \\
C_{O} \frac{d v_{o}}{d t}=\frac{(1-d) i_{L m 1}}{(N+1)}-\frac{v_{o}}{R_{O}}
\end{array}\right.
\end{aligned}
$$

gives the state space equation during $\mathrm{ON}$ and $\mathrm{OFF}$ period. The use of state space averaging method [14] leads to the following set of differential equations that describe the converter dynamics

$$
\left\{\begin{array}{c}
L_{m 1}\left\langle\frac{d i_{L m 1}}{d t}\right\rangle=v_{i n}-d_{s 1}^{\prime} v_{C c 1} \\
L_{m 2}\left\langle\frac{d i_{L m 2}}{d t}\right\rangle=v_{i n}-d_{s 2}^{\prime} v_{C c 2} \\
C_{C 1}\left\langle\frac{d v_{C c 1}}{d t}\right\rangle=i_{L m 1} d_{s 1}^{\prime}-\frac{T_{s}}{2 L_{k}}\left((N+1) v_{C c 1}-\frac{v_{O}}{2}\right) d_{s 1}^{\prime 2} \\
C_{C 2}\left\langle\frac{d v_{C c 2}}{d t}\right\rangle=i_{L m 2} d_{s 2}^{\prime}-\frac{T_{s}}{2 L_{k}}\left((N+1) v_{C c 2}-\frac{v_{O}}{2}\right) d_{s 2}^{\prime 2} \\
C_{O}\left\langle\frac{d v_{O}}{d t}\right\rangle=\frac{\left(d_{s 1}^{\prime}+d^{\prime \prime}\right) i_{L m 1}}{(N+1)}-\frac{v_{O}}{R_{O}}
\end{array}\right.
$$

Where $d_{s 1}, d_{s 2}$ are the duty cycles of main switch $S_{1}$ and $S_{2}$ respectively. $d_{s 1}^{\prime}=\left(1-d_{s 1}\right) d_{s 2}^{\prime}=\left(1-d_{s 2}\right), d^{\prime \prime}$ is the period between the peak value of the leakage inductor current to its zero crossing during the positive half cycle and $T_{S}$ is the switching time.

\section{A. Steady State}

The state space averaged DC model that describe the converter in equilibrium is obtained by letting the left-hand side (LHS) of (11) equal to zero, from which

$$
\mathrm{X}=-A^{-1} B U
$$

And the set of attainable equilibrium points are

$$
\begin{aligned}
& \times=\left[\begin{array}{c}
I_{L m 1} \\
I_{L m 2} \\
V_{C c 1} \\
V_{C c 2} \\
V_{O}
\end{array}\right] \\
& =\left[\begin{array}{c}
\frac{2 T_{s} V_{\text {in }}(N+1)^{2}}{4 L_{k}(N+1)+R_{o} T_{s}\left(1+d^{2}+d^{\prime}-2 d-d d^{\prime}\right)} \\
\frac{2 T_{S} V_{\text {in }}(N+1)^{2}}{4 L_{k}(N+1)+R_{o} T_{s}\left(1+d^{2}+d^{\prime}-2 d-d d^{\prime}\right)} \\
V_{\text {in }} /(1-D) \\
V_{\text {in }} /(1-D) \\
\frac{2 T_{s} V_{\text {in }} R_{o}(N+1)\left(1-d+d^{\prime}\right)}{4 L_{k}(N+1)+R_{o} T_{S}\left(1+d^{2}+d^{\prime}-2 d-d d^{\prime}\right)}
\end{array}\right]
\end{aligned}
$$

From (13), the non-ideal steady state voltage gain expression of the converter is given by

$$
\begin{aligned}
& \frac{V_{O}}{V_{\text {in }}} \\
& =\frac{2 T_{s} R_{o}(N+1)\left(1-d+d^{\prime}\right)}{4 L_{k}(N+1)+R_{o} T_{s}\left(1+d^{2}+d^{\prime}-2 d-d d^{\prime}\right)}
\end{aligned}
$$

The Laplace transfer function of the converter is derived from the state space model using (11) From which the line to output transfer function gives

$$
G(s)=\left|\begin{array}{cc}
S I-A & -B \\
C & D
\end{array}\right| /|S I-A|
$$




$$
G_{v}(s)=\frac{V_{o}}{V_{\text {in }}}(s)=\frac{p_{1} s+p_{2}}{s^{3}+q_{1} s^{2}+q_{2} s+q_{3}}
$$

Where $p_{1}=\left(1-d+d^{\prime}\right) \cdot s /(N+1) C_{o} L_{m}, p_{2}=$ $T_{S}(1-d)^{2}\left(1-d+d^{\prime}\right) / 2 L_{k} C_{c} L_{m}, q_{1}=\frac{T_{S}(1-d)^{2}(N+1)}{2 L_{k} C_{c}}+\frac{1}{R_{o} C_{o}}$ $q_{2}=\frac{T_{s}(1-d)^{2}(N+1)}{2 L_{k} C_{c} R_{o} C_{o}}+\frac{(1-d)^{2}}{L_{m} C_{c}}, \quad q_{3}=\frac{T_{s}(1-d)^{2}\left(1-d+d^{\prime}\right)}{4(N+1) L_{m} L_{k} C_{c} C_{o}}+\frac{(1-d)^{2}}{L_{m} C_{c} R_{o} C_{o}}$

The model in (16) is verified with direct simulation with switch model under full load condition. Fig. shows the simulated and calculated model.

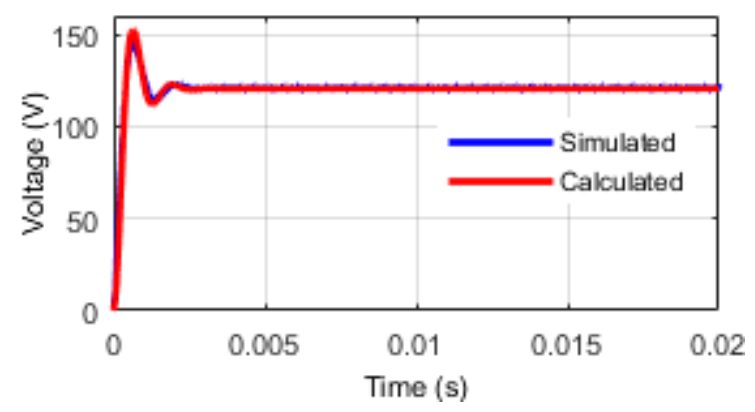

Fig. 2 Switch and calculated model validation

\section{B. Perturbation and Linearization}

A linearized system can be developed by introducing perturbation around the steady state value of the averaged model calculated in (11), containing the steady state dc value represented by uppercase letter and a superimposed ac variation represented by lowercase symbol with circumflex [8-11]. For instance the perturbation definitions for the state variable are: $i_{L m}=I_{L m}+\hat{\imath}_{L m}, v_{i n}=V_{i n}+\hat{v}_{i n}, v_{o}=V_{o}+\hat{v}_{o}$ and $d=$ $D+\hat{d}$. Then by expanding and neglecting the higher order perturbation terms, then removing the steady-state quantities gives the small signal ac model of the converter.

$$
\left\{\begin{array}{c}
L_{m 1} \frac{d\left(I_{L m 1}+\hat{\imath}_{L m 1}\right)}{d t}=V_{i n}+\hat{v}_{i n}-\left(V_{C c 1}+\hat{v}_{C c 1}\right)\left(1-D-\hat{d}_{s 1}\right) \\
L_{m 2} \frac{d\left(I_{L m 2}+\hat{\imath}_{L m 2}\right)}{d t}=V_{i n}+\hat{v}_{i n}-\left(V_{C c 2}+\hat{v}_{C c 2}\right)\left(1-D-\hat{d}_{s 2}\right) \\
C_{C 1} \frac{d\left(V_{C c 1}+\hat{v}_{C c 1}\right)}{d t}=\left(I_{L m 1}+\hat{\imath}_{L m 1}\right)\left(1-D-\hat{d}_{s 1}\right) \\
-\frac{T_{s}\left(1-D-\hat{d}_{s 1}\right)^{2}}{2 L_{k}}\left((N+1)\left(V_{C c 1}+\hat{v}_{C c 1}\right)+\frac{\left(V_{o}+\hat{v}_{o}\right)}{2}\right) \\
C_{C 2} \frac{d\left(V_{C c 2}+\hat{v}_{C c 2}\right)}{d t}=\left(I_{L m 2}+\hat{\imath}_{L m 2}\right)\left(1-D-\hat{d}_{s 2}\right) \\
-\frac{T_{s}\left(1-D-\hat{d}_{s 2}\right)^{2}}{2 L_{k}}\left(\begin{array}{c}
\left.(N+1)\left(V_{C c 2}+\hat{v}_{C c 2}\right)+\frac{\left(V_{o}+\hat{v}_{o}\right)}{2}\right) \\
C_{o}\left\langle\frac{d\left(V_{o}+\hat{v}_{o}\right)}{d t}\right\rangle=\frac{I_{L m 1}+\hat{\imath}_{L m 1}}{(N+1)}\left(1-D-\hat{d}_{s 1}+D^{\prime}+\hat{d}^{\prime}\right) \\
-\frac{\left(V_{o}+\hat{v}_{o}\right)}{R_{o}}
\end{array}\right.
\end{array}\right.
$$

\section{Order Reduction}

Considering a perfect symmetry among the phases of the converter such that all the components are exactly the same, the duty cycle of the main switches are the same and that of corresponding clamp switches are also similar i.e.

$$
\left\{\begin{array}{l}
L_{m 1}=L_{m 2}=L_{m} \\
C_{C 1}=C_{C 2}=C_{C} \\
d_{s 1}=d_{s 2}=d_{s}
\end{array}\right.
$$

In addition, the voltage across clamp capacitors are equal $V_{C c 1}=V_{C c 2}=V_{C c}$ and there is equal current sharing between the converter phases exist such that $i_{L m 1}=i_{L m 2}=i_{L m}, i_{L m 1}+$ $i_{L m 2}=i_{I n}$. When these condition are taking into account the fifth order equation in (17) can be written as

$$
\left\{\begin{array}{c}
L_{m} \frac{d \hat{\imath}_{i n}}{d t}=2 \hat{v}_{i n}-V_{C c} \hat{d}-2(1-D) \hat{v}_{C c} \\
C_{C}\left\langle\frac{d v_{C c}}{d t}\right\rangle=(1-D) \hat{\imath}_{L m}-I_{L m} \hat{d}-\frac{T_{S}(1-D)^{2}}{2 L_{k}} \\
\left((N+1) \hat{v}_{C c}-\frac{\hat{v}_{o}}{2}\right)+\frac{T_{s}(1-D) \hat{d}}{L_{k}}\left((N+1) V_{C c}-\frac{V_{o}}{2}\right) \\
C_{o} \frac{d \hat{v}_{o}}{d t}=\frac{\hat{\imath}_{i n}\left(1-D+D^{\prime}\right)}{2(N+1)}-\frac{I_{L m} \hat{d}}{2(N+1)}+\frac{I_{L m} \hat{d}^{\prime}}{(N+1)}-\frac{\hat{v}_{o}}{R_{o}}
\end{array}\right.
$$

Taking Laplace transform of (19) and replacing $s$ with $d / d t$. Then eliminating $\hat{d}^{\prime}$ yield the small signal lowfrequency model of the interleaved high step-up converter.

$$
\left\{\begin{array}{c}
s \cdot L_{m} \cdot \hat{\imath}_{i n}(s)=2 \hat{v}_{i n}(s)-V_{C c} \hat{d}(s)-2(1-D) \hat{v}_{C c}(s) \\
s \cdot C_{c} \cdot \hat{v}_{C c}(s)=(1-D) \hat{\imath}_{L m}(s)-I_{L m} \hat{d}(s)-\frac{T_{s}(1-D)^{2}}{2 L_{k}} . . \\
\left((N+1) \hat{v}_{C c}(s)-\frac{\hat{v}_{o}(s)}{2}\right)+\frac{T_{s}(1-D) \hat{d}(s)}{L_{k}}\left((N+1) V_{C c}-\frac{V_{o}}{2}\right) \\
s C_{o} \hat{v}_{o}(s)=\frac{\hat{\imath}_{i n}(s)\left(1-D+D^{\prime}\right)}{2(N+1)}-\frac{I_{L m} \hat{d}(s)}{2(N+1)}+\frac{I_{L m} \hat{d}^{\prime}(s)}{(N+1)}-\frac{\hat{v}_{o}(s)}{R_{o}}
\end{array}\right.
$$

From (19) the system matrix can be written as A

$$
\begin{gathered}
=\left[\begin{array}{ccc}
s & \frac{2(1-D)}{L_{m}} & 0 \\
-\frac{(1-D)}{C_{c}} & s+\frac{(N+1)(1-D)^{2}}{2 f_{s} L_{k} C_{c}} & -\frac{(1-D)^{2}}{4 L_{k} C_{c}} \\
-\frac{\left(1-D+D^{\prime}\right)}{2(N+1) C_{o}} & \frac{2 I_{L m}(1-D)}{C_{o} V_{o}} & s+\frac{1}{R_{o} C_{o}}+\frac{2 I_{L m}\left(1-D+D^{\prime}\right)}{(N+1) C_{o} V_{o}}
\end{array}\right] \\
B=\left[\begin{array}{ccc}
\frac{2 V_{C c}}{L_{m}}, & \frac{(1-D)\left((N+1) V_{C c}-\frac{V_{o}}{2}\right)}{f_{S} L_{k} C_{c}}-\frac{I_{L m}}{C_{c}}, & -\frac{2 I_{L m} V_{C c}}{V_{o} C_{o}}
\end{array}\right]^{T}, \quad C=\left(\begin{array}{l}
0 \\
0 \\
1
\end{array}\right)
\end{gathered}
$$

Following this various transfer functions can be derived suitable for closed loop control system design of the converter

$$
\left[\begin{array}{c}
\hat{\imath}_{\text {in }}(s) \\
\hat{v}_{C C}(s) \\
\hat{v}_{o}(s)
\end{array}\right]=[A(s)]^{-1}\left[\begin{array}{c}
b_{1}(s) \\
b_{2}(s) \\
b_{3}(s)
\end{array}\right] \hat{d}(s)+[A(s)]^{-1}[C(s)] \hat{v}_{i n}(s)
$$

\section{Duty Ratio Control}

Direct duty ratio control is obtained by making $\hat{v}_{\text {in }}(s)=0$ in (21) given by

$$
G_{v d}(s)=\frac{\hat{v}_{o}(s)}{\hat{d}(s)}=\frac{a_{1} s^{2}+a_{2} s+a_{3}}{p_{1} s^{3}+p_{2} s^{2}+p_{3} s+p_{4}}
$$

Where $a_{1}=L_{m} C_{c} b_{1}, a_{2}=\frac{\left(1-D+d^{\prime}\right) c_{c}}{2(N+1)} \cdot b_{1}+\frac{2 I_{L m} L_{m}(1-D)}{V_{o}} \cdot b_{2}+\frac{(1-D)^{2} L_{m}}{f_{s} L_{K}} \cdot b_{3}$ $a_{3}=(1-D)^{2}\left(\frac{\left(1-D+D^{\prime}\right)}{2 f_{s} L_{K}}+\frac{2 I_{L m}(1-D)}{V_{o}}\right) \cdot b_{1}-\frac{2\left(1-D+D^{\prime}\right)(1-D)}{(N+1)} \cdot b_{2}+$ 
$2(1-D)^{2} \cdot b_{3},, p_{2}=\frac{L_{m} C_{O}(1-D)^{2}}{f_{s} L_{K}}+L_{m} C_{c}\left(\frac{1}{R_{O}}+\frac{2 I_{L m}\left(1-D+D^{\prime}\right)}{(N+1) V_{o}}\right)$,

$p_{1}=L_{m} C_{c} C_{O}, p_{3}=\frac{L_{m}(1-D)^{2}}{f_{s} L_{K}}\left(\frac{1}{R_{O}}+\frac{2 I_{L m}\left(1-D+D^{\prime}\right)}{(N+1) V_{o}}\right)+2 C_{O}(1-D)^{2}$,

$p_{4}=2(1-D)^{2}\left(\frac{1}{R_{O}}+\frac{2 I_{L m}\left(1-D+D^{\prime}\right)}{(N+1) V_{o}}+\frac{(1-D)\left(1-D+D^{\prime}\right)}{f_{S} L_{K}(N+1)^{2}}\right)$

Where $D^{\prime}$ represents the steady state dc value of $d^{\prime}$.

The control-to-output transfer function describes a standard third order system and has a negative real pole at $s=$ $-5.65 e 05$, two complex conjugate poles at $s=-7.57 \pm$ $j 1.3 e 03$, and two zeros at $s=-5.65 e 05$ and $s=1.34 e 04$ respectively. The control-to-output transfer function exhibit a non-minimum phase system, which is typical behaviour of converters with boost or buck-boost characteristics.

The control-to-output transfer function in (22) is once again verified in simulation by perturbing duty cycle set point with sinusoids of different frequencies and stores the corresponding output voltage. A discrete points are obtained from the frequency response that describes how the system responds to the magnitude and phase of the injected sinusoids. In essence, the control-to-output transfer function can be estimated from the measured data. Fig. 3 illustrates the Bode plot of both the calculated and estimated response of the control-to-output transfer function. As can be seen a good agreement exist between the models and the calculated model is suitable for the frequency domain analysis and controller design. The parameters of the simulation and the calculated model are listed in Table I.

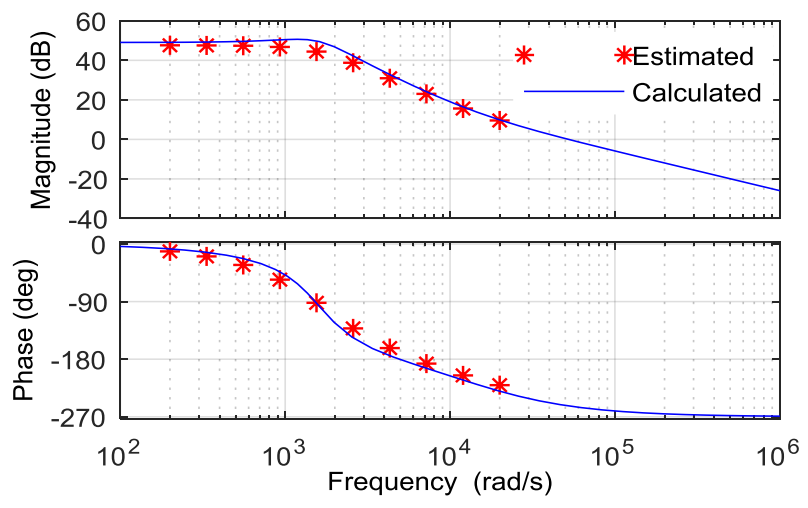

Fig. 3 Calculated and estimated control to output transfer function

TABLE I. CONVERTER PARAMETERS

\begin{tabular}{ll}
\hline \hline \multicolumn{1}{c}{ Parameter } & \multicolumn{1}{c}{ Rating } \\
\hline Output Power $\left(P_{O}\right)$ & $500 \mathrm{~W}$ \\
Input Voltage $\left(V_{\text {in }}\right)$ & $12 \mathrm{~V}$ \\
Output Voltage $\left(V_{O}\right)$ & $120 \mathrm{~V}$ \\
Switching Frequency $\left(f_{s}\right)$ & $50 \mathrm{KHz}$ \\
Clamp capacitors $\left(C_{C}\right)$ & $4.7 \mu \mathrm{F}$ \\
Switched Capacitor $\left(C_{m}\right)$ & $10 \mu \mathrm{F}$ \\
Output capacitor $\left(C_{O}\right)$ & $50 \mu \mathrm{F}$ \\
Turns Ratio $\left(n_{2} / n_{1}\right)$ & $1: 1$ \\
Magnetizing Inductance $\left(L_{m}\right)$ & $36 \mu \mathrm{H}$ \\
\hline \hline
\end{tabular}

\section{CONTROLler DESIGN}

Fig. 4 illustrates the block diagram of the control strategy used to investigate the closed loop dynamic performance of the converter. It is a typical dual loop control, comprising of inner current loop and outer voltage loop. The presence of the right half plane (RHP) zero in the control-to-output transfer function of (21) tends to destabilize the single-loop feedback control. It is difficult to obtain an adequate phase margin (PM), because during transient the phase lag of the right (RHP) zero causes the output to change initially in the wrong direction [12]. The loops are usually defined to satisfy certain design criteria of PM and bandwidth.

The feedback control system uses proportional-integral (PI) controller in both loops. The outer voltage loop determines the current reference of the inner current loop, whilst the control signal is determined by the inner current controller. The control signal generates the gating signals of the main switches $S_{1}$ and $S_{2}$ with the same duty ratio with the aid of digital pulse width modulation (DPWM) submodule. Two modulators shifted in phase by $180^{\circ}$ are used to produce the gating signals. Note that the gate signal of the clamp switches $S_{C 1}$ and $S_{C 2}$ are complementary to their corresponding primary switches. The current and voltage transducers have been taken into account with their respective gains $H_{i}, H_{v}$.

The outer voltage loop has slow dynamics whilst the inner current loop has fast dynamics. This is to allow the input current to respond more quickly than the converter output voltage. The DPWM module in Fig. 5 comprises of the Padè approximation block and the moldulator static gain [12].

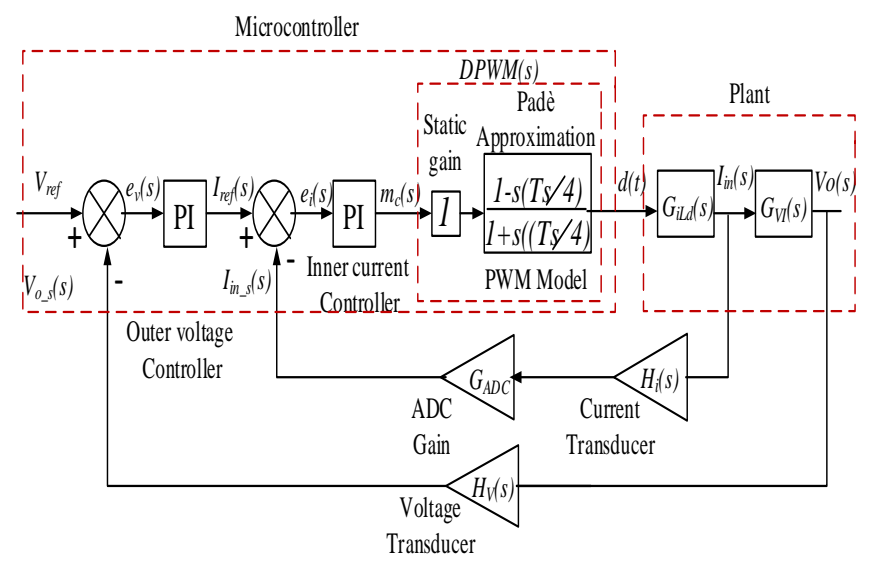

Fig. 4 Block diagram of current mode control

\section{A. Inner Current Control Loop}

From Fig. 4, the inner current PI controller is designed first, using the control to input current transfer function from (23)

$$
G_{i d}(s)=\frac{\hat{i}_{i n}(s)}{\widehat{d}(s)}=\frac{q_{1} s^{2}+q_{2} s+q_{3}}{p_{1} s^{3}+p_{2} s^{2}+p_{3} s+p_{4}}
$$

Where

$$
\begin{aligned}
& q_{1}=b_{1} \cdot s^{2}+\left(\frac{1}{R_{O} C_{O}}+\frac{(N+1)(1-D)^{2}}{2 f_{S} L_{K} C_{c}}+\frac{2 I_{L m}\left(1-D+D^{\prime}\right)}{(N+1) V_{o}}\right) \cdot b_{2} s \\
& +\frac{(N+1)(1-D)^{2} \cdot b_{3}}{2 f_{S} L_{K} C_{c}}\left(\frac{1}{R_{O} C_{O}}+\frac{2 I_{L m}\left(1-D+D^{\prime}\right)}{(N+1) V_{o}}\right)+\frac{2 I_{L m}(1-D)^{3}}{4 f_{s} L_{k} C_{c} C_{o} V_{o}} \cdot b_{3} \\
& q_{2}=-2(1-D) C_{o} \cdot b_{1}-2(1-d)\left(\frac{1}{R_{O}}+\frac{2 I_{L m}\left(1-D+D^{\prime}\right)}{(N+1) V_{o}}\right) \cdot b_{2} \\
& q_{3}=-\frac{(1-D)^{3}}{(N+1) f_{S} L_{K}} \cdot b_{3}
\end{aligned}
$$

Following this, the open loop transfer function of the inner current control loop (simply a cascade connection of all blocks) 
is required to design the controller. This transfer function is given by

$$
\begin{aligned}
& G_{o L}(s) \\
& =\frac{K_{p}\left(s+K_{i} / K_{p}\right)}{s} \cdot \frac{\left(1-s T_{s} / 4\right)}{\left(1+s T_{s} / 4\right)} \cdot H_{i}(s) \cdot G_{i d}(s)
\end{aligned}
$$

Where $H_{i}(s)$ is the current sensor gain. The continuous time system in (24) is first discretized with zero order hold $(\mathrm{ZOH})$ given by

$$
G_{i d}(z)=Z\left\{\frac{1}{s}\left(1-e^{-s T s}\right) H(s) \cdot G_{i d}(s)\right\}
$$

Once this is available, the digital PI controller is designed directly in the discrete time domain using methods similar to continuous time frequency response. The compensator design is driven by certain specifications concerning the closed loop performance (such as speed of response or tracking error with respect to the reference signal). For this reason, a closed loop bandwidth $f_{C L}$ of one tenth of the switching frequency $f_{s}$ is intended to be achieved with at least PM of $60^{\circ}$. The subsequent step is to determine the proportional gain $K_{p}$ and integral gain $K_{I}$ that guarantee compliance with these specifications. Fig. 5 illustrate the Bode diagram of the inner

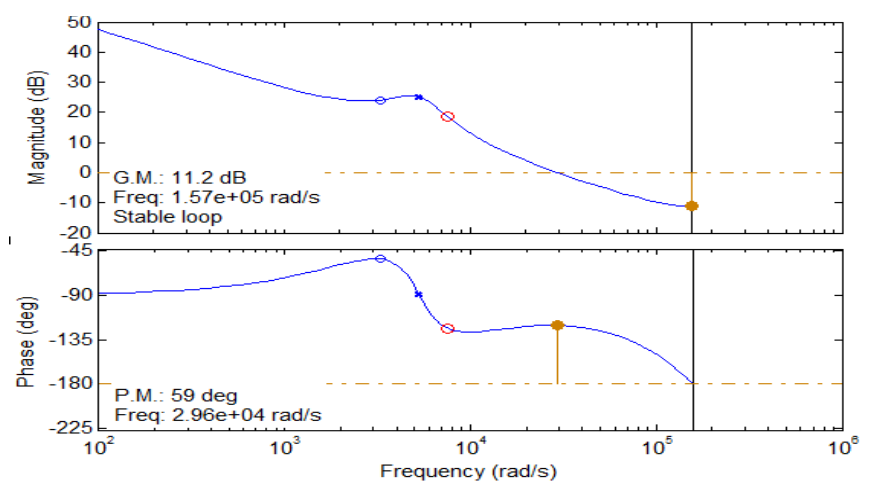

Fig. 5 Control to duty cycle transfer function Bode diagram

current loop, showing compliance with design specifications. The controller is designed in Matlab using a "sisotool" graphical user interface based on Zeigler-Nichols tuning that allows the closed loop frequency response to be interactively changed by modifying the pole-zero location of the PI compensator. The corresponding feedback compensator gains from the same interface are $K_{p}=0.0151$ and $K_{I} T_{s}=0.0025$ respectively.

\section{B. Outer Voltage Loop}

The outer voltage PI controller is design in similar way using the voltage to current transfer function (26)

$$
G_{v i}(s)=\frac{\hat{v}_{o}(s)}{\hat{I}_{i n}(s)}=\frac{a_{1} s^{2}+a_{2} s+a_{3}}{q_{1} s^{2}+q_{2} s+q_{3}}
$$

Where $a_{1}=L_{m} C_{c} b_{1}, a_{2}=\frac{\left(1-D+d^{\prime}\right) c_{c}}{2(N+1)} \cdot b_{1}+\frac{2 I_{L m} L_{m}(1-D)}{V_{o}} \cdot b_{2}+\frac{(1-D)^{2} L_{m}}{f_{s} L_{K}} \cdot b_{3}$ $a_{3}=(1-D)^{2}\left(\frac{\left(1-D+D^{\prime}\right)}{2 f_{s} L_{K}}+\frac{2 I_{L m}(1-D)}{V_{o}}\right) \cdot b_{1}-\frac{2\left(1-D+D^{\prime}\right)(1-D)}{(N+1)} \cdot b_{2}+2(1-$ $D)^{2} \cdot b_{3}, \quad q_{1}=b_{1} \cdot s^{2}+\left(\frac{1}{R_{O} C_{O}}+\frac{(N+1)(1-D)^{2}}{2 f_{S} L_{K} C_{C}}+\frac{2 I_{L m}\left(1-D+D^{\prime}\right)}{(N+1) V_{O}}\right) \cdot b_{2} s+$ $\frac{(N+1)(1-D)^{2} \cdot b_{3}}{2 f_{S} L_{K} C_{C}}\left(\frac{1}{R_{O} C_{O}}+\frac{2 I_{L m}\left(1-D+D^{\prime}\right)}{(N+1) V_{O}}\right)+\frac{2 I_{L m}(1-D)^{3}}{4 f_{S} L_{k} C_{C} C_{o} V_{o}} \cdot b_{3}, q_{3}=-\frac{(1-D)^{3}}{(N+1) f_{S} L_{K}} \cdot b_{3}$
$q_{2}=-2(1-D) C o \cdot b_{1}-2(1-d)\left(\frac{1}{R_{O}}+\frac{2 I_{L m}\left(1-D+D^{\prime}\right)}{(N+1) V_{o}}\right) \cdot b_{2}$,

The open loop gain is given by

$$
G_{o L}(s)=\frac{K_{p}\left(s+K_{i} / K_{p}\right)}{s} \cdot H_{v}(s) \cdot G_{v i}(s)
$$

Where $H_{v}(s)$ is the voltage transducer gain. To ensure sufficient stability around equilibrium point due to parameter variation influence, a closed loop bandwidth $f_{C L}$ of one tenth of the inner current loop is intended to be achieved with at least phase

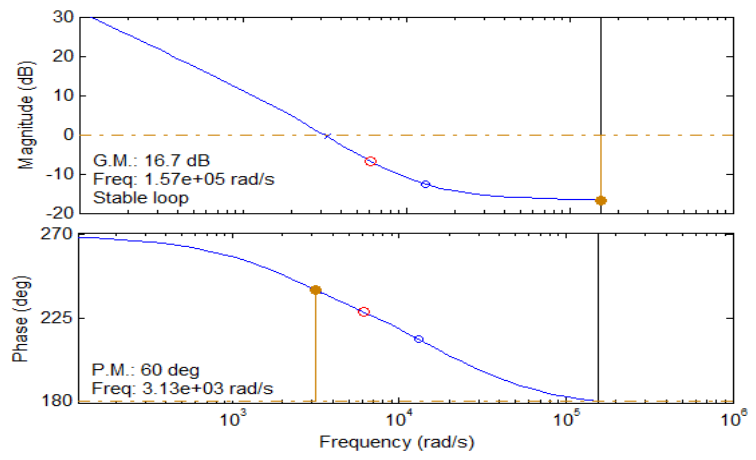

Fig. 6 Bode diagram of voltage control loop with discrete PI controller

margin PM of $60^{\circ}$. Following the same method described in the current loop the proportional gain $K_{p}$ and integral gain $K_{I}$ of the voltage loop that guarantees compliance with these specifications are $K_{p}=0.1987$ and $K_{I} T_{s}=0.00258$ respectively. Fig. 6 shows the Bode plot of the voltage control loop with the discrete controller. The desired specifications of phase and gain margins were achieved and the low-frequency gain is improved.

\section{Simulation Results}

To verify the theoretical analysis and the closed loop dynamics performance of the converter. A $500 \mathrm{~W}$ is designed and built in Matlab/Simulink with the parameters listed in Table I. The transient response characteristics such as settling time, peak over shoot and steady state error for output voltage regulation during the load perturbation are observed. Fig. 7 and Fig. 8 shows the simulated response of the converter due to load disturbances.

In Fig. 7, a step change in load resistance is applied, causing a step decrement in output power from $500 \mathrm{~W}$ to $100 \mathrm{~W}$ and vice versa. The peak overshoot is $20 \%$ of the steady state output voltage and the settling time is $30 \mathrm{~ms}$. Another load disturbance is applied in Fig. 8, causing a step decrement in output power from $250 \mathrm{~W}$ to $400 \mathrm{~W}$ and vice versa. Unlike the previous case, the output response settle faster within $15 \mathrm{~ms}$ and the overshoot/undershoot reduces to $6.6 \%$ of the steady state values

\section{CONCLUSION}

The derivation of the reduced order small signal model of interleaved high step-up converter with coupled inductors and switched capacitor in CCM is presented in this paper. The validity of the derived model is verified in simulation and used in the design of the dual loop controllers. The dynamic response of the converter under the influence of the designed PI 

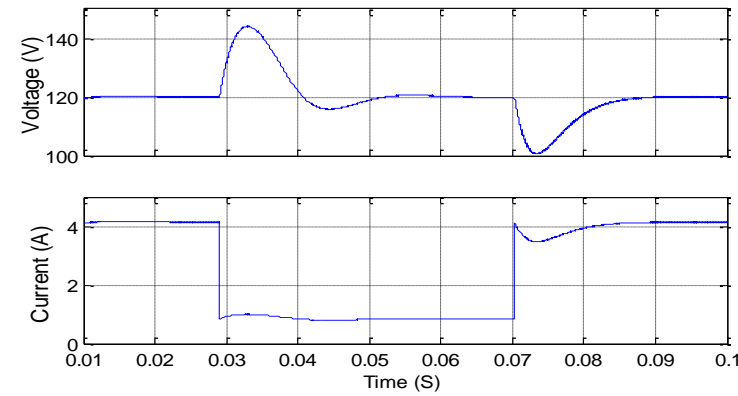

Fig. 7 Step change in load from full load down to $20 \%$ load

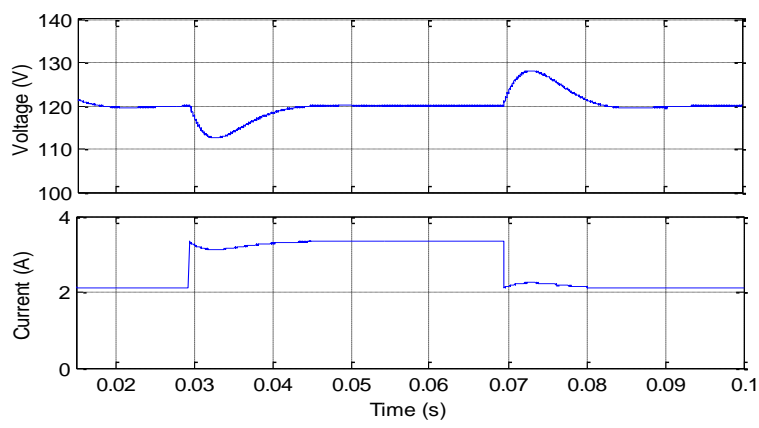

Fig. 8 Load change from half load to $80 \%$ load

controllers during load disturbance is also verified via a simulation. The proposed reduced order model exhibits good transient response during disturbances and adequate for controller design capable of good voltage regulation and disturbance rejection.

\section{REFERENCES}

[1] M. Muhammad, M. Armstrong, and M.A. Elgendy. Modelling and control of non-isolated high voltage gain boost converter employing coupled inductor and switched capacitor. in 2016 International Conference for Students on Applied Engineering (ICSAE). 2016
[2] F.S. Garcia, J. S. Pomilio and G. Spiazzi., "Modeling and control design of the six-phase Interleaved Double Dual Boost converter." IEEE Trans. on Ind. Electrons,, 2013. 60(8): p. 3283-3290.

[3] Y. J. A. Alcazar, W. G. C. Cabero, R. P. Bascop'e, S. Daher, D. S OliveiraJr., and G. J. M. de Sousa, "Modeling and control of the high voltage gainboost converter based on three-state switching cell and voltage multipliers (mc)," in Proc. Brazil. Power Electron. Conf., Sep./Oct., pp. 655-664.

[4] Y.H. Liao, C.-M. Lai, and Y.-L. Ke, "Modeling and design of an improved current-fed converter with new voltage multiplier circuit combination," in Proc. IEEE Ind. Appl. Soc. Annu. Meet., Oct. 2011, pp. $1-8$.

[5] J. C. Mayo-Maldonado, R. Salas-Cabrera, J. C. Rosas-Caro, J. De LeonMorales, and E. N. Salas-Cabrera, "Modelling and control of a DC-DC multilevel boost converter," IET Power Electron., vol. 4, no. 6, pp. 693$700,2011$.

[6] Muhammad, M., M. Armstrong, and M. Elgendy, "A Non-isolated Interleaved Boost Converter for High Voltage Gain Applications". IEEE Journal of Emerging and Selected Topics in Power Electronics, vol. 4 no 2, pp. 352-362, Jun. 2016

[7] Muhammad, M., M. Armstrong, and M. Elgendy, "Analysis and implementation of high-gain non-isolated DC-DC boost converter". IET Power Electron, 201710 (11), 1241-1249

[8] G. Spiazzi., S. Buso, F. Sichirollo, and L. Corradini "Small-signal modelling of the interleaved boost with voltage multiplier" In IEEE Energy Conversion and Exposition (ECCE), 2012. p. 431-437.

[9] F.H. Dupont, C. Rech, R. Gules, J.R Pinheiro., "Reduced-Order Model and Control Approach for the Boost Converter With a Voltage Multiplier Cell." IEEE Transactions on Power Electronics,, 2013. 28(7): p. 3395 3404.

[10] R. Carlos, J. Calvente, A. Romero,R. Giral, "Current-Mode Control of a Coupled-Inductor Buck Boost DC-DC Switching Converter." IEEE Transactions on Power Electronics,, 2012. 27(5): p. 2536-2549.

[11] R. W. Erickson and D. Maskimovic, Fundamentals of Power Electronics. USA: Springer, 2000.

[12] S. Buso and P.Matavelli, Digital Control in Power Electronics. USA: Morgan \& Claypool, 2006 